\section{PLTLIB: A FORTRAN plot library for the IEEE 488-1975 general-purpose instrument bus}

\section{JOSEPH J. GRUHN \\ Veterans Administration Medical Center, Cleveland, Ohio}

The Digital Equipment Corporation (DEC) has available the IBLIB instrument-bus library, which supports either the IBV11-A or IB11 instrument-bus interface between a DEC PDP.11 system and the IEEE 488-1975 general-purpose instrument bus (GPIB). However, use of the library exclusively for interfacing with a specific device such as the Tektronix 4662 digital plotter is an inefficient and time-consuming task for the programmer. An easy to use, FORTRAN-callable subroutine library (PLTLIB) has been developed to utilize IBLIB efficiently and to specifically perform the functions most commonly desired from the 4662 plotter.

In addition to PLTLIB, another subroutine (PLOT3D) and main program (ANYPLT) were developed to exemplify the use of the library. PLOT3D is a useful FORTRAN subroutine for plotting three-dimensional surfaces, and ANYPLT is an interactive program that can plot up to 18 data curves of any number of points on a standard X.Y data plane.

Description. The PLTLIB library contains 37 FORTRAN-callable subroutines, 16 of which are the standard 4662 GPIB plotter commands. These include: five subroutines that manipulate the alphanumerics of the digital plotter; three subroutines that position the plotter pen; one subroutine that draws a line to an absolute GDU (graphic-display unit) position; four listening subroutines that receive information from the plotter; a setstatus word and a prompt light (on/off) routine. In addition to these standard commands, 21 subroutines have been written to aid in the usual use of the 4662 plotter (e.g., plotting data curves and drawing figures, charts, and diagrams). These additional subroutines fall into four general categories: plotter inactive "set-up" functions; move and draws; ASCII print routines; and figure routines.

The plotter inactive functions are VIE, WIN, INI, ZUM, and CLP. VIE is a viewporting request that adjusts the plotting field of the 4662 to any rectangular portion of the total plotting surface. WIN is a window request that then adjusts the X-Y ranges of the plotting field to accommodate any specific task. All subsequent values passed to a PLTLIB routine should be within the range of the $X-Y$ window last requested, or else the data will be clipped at the viewpoint edges. The values passed are

The author's mailing address is: Psychophysiological Research Laboratory, Veteran's Administration Medical Center, Brecksville, Ohio 44141. then adjusted by the PLTLIB into GDUs and sent to the plotter. The routine INI initializes the plotter to default X-Y window ranges of 0 to 150 and 0 to 100 , respectively, and selects a viewport that uses the entire plotting surface. INI also resets any alpharotate, alpha-scale, or font specifications to the plotter default values. The routine ZUM allows the programmer to zoom a plot display to a proportion of the original plot without any alteration of window and viewport specifications. Another routine, CLP, allows the programmer to set an override switch for viewport clipping. This then allows the programmer to move and draw outside the requested window.

There are six move and draw routines. Two routines, RMO and RDR, allow the programmer to pass relative values for a move or draw from a current pen position to an $\mathrm{X}$ and $\mathrm{Y}$ distance requested in the call. Two more routines, MOV and DRA, require the programmer to pass an absolute X-Y point within the specified window, and cause the plotter to either move or draw from the current pen position to the requested point. The routines DSD and DSH draw a dash-dot, or a dashed line of variable increments, to an absolute point requested.

Two ASCII print routines, PRN and PRX, were developed in addition to $P R I$, a routine that utilizes a standard GPIB command to instruct the plotter to print an ASCII character string. PRN allows the programmer to pass an integer value, and PRX accepts a floatingpoint value. Both routines convert the passed value to an ASCII character string and cause the plotter to print the encoded value as a character string at its current pen position.

There are eight figure routines that cause multiple moves and draws by the plotter. Six of these, TRI, BOX, CIR, EXX, PLU, and DOT, draw fixed figures of variable GDU sizes centered at an absolute point passed by the calling routine. Another routine, AXI, draws a specific $X-Y$ axis with variable increment hashmarks. Finally the routine FIG draws a polygon of any number of lines.

A complete summary listing of all the PLTLIB routines, their arguments, and their functions is in Table 1.

The PLOT3D subroutine utilizes the PLTLIB library to plot a three-dimensional graph of up to $128 \times 128$ in size. The user of PLOT3D must pass the two-dimensional array of values, the dimension of the array, the Xaxis, the $Y$-axis, and the $Z$-axis viewing distances from the origin, and the angle of rotation about a specified $X$ and $Y$ point. The data array must be in integer form and be stored in virtual memory to conserve space. PLOT3D does not use a hidden-lines algorithm and is currently limited to rotation about the Z-plane. The actual calculation of the three-dimensional graph takes about 2.5 min for a $128 \times 128$ display; however, actual plotting time for a cross-hatched plot is close to $40 \mathrm{~min}$, due to the limited speed of the 4662 pen movement. 
Table 1

A Summary of PLTLIB FORTRAN-Callable Subroutines, the Arguments Passed, and the Routine Functions

\begin{tabular}{|c|c|c|}
\hline Routine & Arguments & Function \\
\hline \multicolumn{3}{|c|}{ Standard GPIB Command Functions } \\
\hline ALF & (IFONT) & Request character font \\
\hline ARE & none & Alpha reset request \\
\hline ART & (ANGLE) & Alpha rotate request \\
\hline ASC & (XSCALE,YSCALE) & Alpha scale request \\
\hline PRI & (STRING) & Print character string \\
\hline CGI & $(\mathrm{X}, \mathrm{Y}, \mathrm{IZ})$ & Call Gin (call button) \\
\hline GIN & $(\mathrm{X}, \mathrm{Y}, \mathrm{IZ})$ & Gin of $X, Y$ and pen up/down \\
\hline HOM & none & Position pen home \\
\hline PAG & none & Position pen home \\
\hline PRO & $(1$ or 0$)$ & Prompt light on/off \\
\hline RES & none & Reset plotter \\
\hline RSW & (ILOC,IVAL) & Read status word \\
\hline SIZ & $(\mathrm{IX}, \mathrm{IY}, \mathrm{ID})$ & Poll for $\mathrm{X}$ and $\mathrm{Y}$ size of plotter and identification word \\
\hline SSW & (ILOC,IVAL) & Set status word \\
\hline MOVE & $(\mathrm{X}, \mathrm{Y})$ & Absolute (GDU) move \\
\hline DRAW & $(\mathrm{X}, \mathrm{Y})$ & Absolute (GDU) draw \\
\hline \multicolumn{3}{|c|}{ Additional Plot Routines } \\
\hline INI & none & Initialize default viewport and window $(0,150,0,100)$ \\
\hline VIE & (XL,XH,YL,YH) & Sets up plotter viewport \\
\hline WIN & $(\mathrm{XL}, \mathrm{XH}, \mathrm{YL}, \mathrm{YH})$ & Sets up plotter window \\
\hline CLP & (LCLIP) & Sets line clipping or no \\
\hline ZUM & (ZOOM) & Zooms portion of display \\
\hline RDR & $(\mathrm{X}, \mathrm{Y})$ & Relative $\mathrm{x}, \mathrm{y}$ draw \\
\hline RMO & $(\mathrm{X}, \mathrm{Y})$ & Relative $x, y$ move \\
\hline DRA & $(\mathrm{X}, \mathrm{Y})$ & Absolute $x, y$ draw \\
\hline MOV & $(\mathrm{X}, \mathrm{Y})$ & Absolute $x, y$ move \\
\hline DSD & $(\mathrm{X}, \mathrm{Y}, \mathrm{INC})$ & Draws a dashed-dotted line \\
\hline DSH & $(\mathrm{X}, \mathrm{Y}, \mathrm{INC})$ & Draws a dashed line \\
\hline PRN & (IVAL) & Prints an integer value \\
\hline PRX & (XVAL) & Prints a floating point value \\
\hline TRI & (X,Y,ISIZE) & Draws a triangle \\
\hline BOX & (X,Y,ISIZE) & Draws a box \\
\hline CIR & (X,Y,ISIZE) & Draws a circle \\
\hline $\mathrm{EXX}$ & $(\mathrm{X}, \mathrm{Y}, \mathrm{ISIZE})$ & Draws a X \\
\hline PLU & $(\mathrm{X}, \mathrm{Y}, \mathrm{ISIZE})$ & Draws a plus sign $(+)$ \\
\hline DOT & $(\mathrm{X}, \mathrm{Y}, \mathrm{ISIZE})$ & Draws a filled circle \\
\hline AXI & (X, Y, XINC, YINC, XRANG, YRANG) & Draws an axis \\
\hline FIG & (XARRAY, YARRAY, IDIMEN) & Draws a polygon of IDIMEN lines \\
\hline
\end{tabular}

The program ANYPLT utilizes the PLTLIB library to make standard data plots of up to 18 lines, each with a different legend. It is an interactive program that is easily run by an inexperienced computer user. The plot is drawn interactively with the user, first with axis and axis labels being specified and plotted and then with data for each line to be graphed being entered by the user and plotted by the 4662 . For each line plotted, the program requests a name and keeps a legend of symbols and line types along with the assigned name to the right of the graph. Finally, the program requests a title and centers it at the top of the graph.

Equipment and Software. The PLTLIB library, PLOT 3D subroutine, and ANYPLT are written in FORTRAN IV for a DEC RT-11 operating system. The software library IBLIB provided by the Digital Equipment Corporation for the interface of either the IBV11-A or IB11 instrument bus with the IEEE 488-1975 general-purpose instrument bus must be concatenated with PLTLIB. A
Tektronix 4662 digital plotter is interfaced through the GPIB with the main computer.

Although the library is written specifically for the Tektronix 4662 , any digital plotter with a GPIB interface should be able to utilize PLTLIB with only minor modifications. The PLTLIB communicates with the plotter through the GPIB by passing byte arrays containing a primary address, secondary address command, and an ASCII character string containing the information the plotter needs to execute the command. A simple change of one data statement in each of the 16 primary plot routines in PLTLIB to accommodate the addressing characteristics of a specific plotter should be the only modification necessary.

Restrictions. Since the routines of PLTLIB belong to a library object file, and none of the routines requires much memory or execution time, the major restriction in the use of the library depends on the size of the calling program and on the memory capacity of the specific 
system used. PLOT3D makes use of virtual memory space that many systems may not have available; however, with slight modification of the program and some loss in execution time, PLOT3D should be easily adaptable to any size system. The program ANYPLT requires very little memory and should easily run on most FORTRAN IV systems without adaptation.

Availability. Complete source listings of PLTLIB, PLOT3D, and ANYPLT, along with a copy of this paper and a text description of all the callable library routines, arguments passed, and routine functions, and along with instructions on the building of PLTLIB and its concatenation with IBLIB, are available free of charge from Joseph J. Gruhn, Psychophysiological Research Laboratory 151(B), Veterans Administration Medical Center, Brecksville, Ohio 44141.

(Manuscript accepted for publication June 6, 1983.) 